\title{
Massively Parallel Detection for Non-Orthogonal Signal Transmissions
}

\author{
Chathura Jayawardena and Konstantinos Nikitopoulos \\ 5G Innovation Center \\ Institute for Communication Systems (ICS) \\ University of Surrey, Guildford, UK \\ Email: \{c.jayawardena, k.nikitopoulos\}@ surrey.ac.uk
}

\begin{abstract}
The increasing demand for massive connectivity with low latency requirements has triggered a paradigm shift towards Non-Orthogonal transmissions. Still, to translate the theoretical gains of Non-Orthogonal transmissions into practical, efficient "soft" detection schemes are required. The detection latency and/or complexity of state-of-the-art detection methods becomes impractical for large Non-Orthogonal systems, both due to the large number of interfering streams and due to the rank-deficient or ill-determined nature of the corresponding interference matrix. Extending the recently proposed MultiSphere framework, this work introduces NorthCore; a massively parallel sphere-decoding-based scheme for the detection of large and illdetermined Non-Orthogonal systems. Similarly to MultiSphere, NorthCore reduces the corresponding search space by focusing the available processing power to the most promising vector solutions that are processed in parallel. As a result, the proposed detection scheme can attain a detection processing latency similar to that of highly-suboptimal linear detectors and even outperform state-of-the-art sophisticated detection approaches with up to an order of magnitude reduced complexity. To identify the most promising vector solutions, NorthCore introduces a sortfree candidate selection technique that reduces the necessary preprocessing complexity by up to an order of magnitude, making the proposed approach practical.
\end{abstract}

Index Terms-Sphere Decoding, Non-Orthogonal Transmission, Parallel Processing

\section{INTRODUCTION}

The next generation of communication systems are expected to provide massive connectivity with low latency requirements. These requirements have introduced a paradigm shift towards Non-Orthogonal transmission schemes. In this context, multicarrier, code-domain Non-Orthogonal multiple access (NOMA) schemes such as LDS-OFDM and SCMA have been of recent research interest [1], [2], mainly for uplink transmissions. In another context, Non-Orthogonal faster-thanNyquist and Spectrally Efficient FDM (SEFDM) sacrifice signal orthogonality to achieve increased throughput and spectral efficiency [3], [4], [5].

However, to deliver these theoretical gains in practice, efficient schemes to demultiplex a large number of mutually interfering streams are necessary. In addition to the high dimensionality of such a detection problem, the interference matrix of recently proposed Non-Orthogonal transmission schemes is either ill-determined (e.g., SEFDM) or even rank-deficient (e.g., LDS-OFDM, power domain NOMA). To demultiplex the corresponding information streams, recently proposed NOMA solutions such as LDS-OFDM and SCMA employ sparse signal transmissions, that enables efficient detection by means of the Message Passing Algorithm (MPA) [6]. Still, the computation complexity of the corresponding messages (per iteration) is determined by the number of mutually interfering streams and the modulation order. In addition, a high number of iterations are necessary to obtain accurate soft information for high order modulation schemes or codebooks as we will show in Section V. Furthermore, MPA does not apply to nonsparse structures such as simple power domain-NOMA [7], MIMO or SEFDM.

For non-sparse signals Sphere Decoding (SD) and its softoutput versions have been introduced as methods to reduce the complexity of Max-Log MAP detection [8], [9]. However, the latency requirements for obtaining exact Max-Log MAP soft information using depth first SDs [10] are random and become impractical even for full rank high dimensional systems. In a similar manner, the complexity of existing approximate fixed latency SD schemes, such as the Soft Fixed Complexity SD (SFSD) [11] and the K-Best list SD [12], does not scale efficiently for large rank-deficient systems and their processing complexity becomes impractical. This is because, in principle, such approaches do not account for the specific interference matrix realization, but target the worst case transmission condition. As a result, list based approaches such as the KBest SD require large $\mathrm{K}$ values, and extensive long sorting operations that compromise their implementation efficiency.

In SEFDM systems, approximate SD based detection schemes have been adopted together with tailored preprocessing schemes that mild the complexity increase introduced by the corresponding ill-conditioned interference matrix. In this direction, the Truncated Singular Value Decomposition (TSVD) [13] has been applied as a preprocessing stage for approximate SDs. This approach, however, sacrifices optimality and results in a irreversable performance loss. The hard SD method together with the iterative preprocessing in [14] does not sacrifice optimality, but results in impractical complexity requirements for higher bandwidth compression, dense constellations and/or fading channels. To further improve the achievable throughput the authors of [15] introduced an iterative soft detection approach that is of very low complexity, but performs poorly for dense constellations.

An ideal detection scheme should be generic and applicable 
to any kind of Non-Orthogonal system employing both sparse and non-sparse signals [16], while efficiently mitigating the deficient or ill-determined rank nature of the interference matrix. In addition, such a detection scheme must be of very low latency and complexity even for a large number of NonOrthogonal streams, to cope with the requirements of stateof-the-art systems [17]. Still, the processing requirements of existing detection approaches can easily exceed the capabilities of traditional processors [18], preventing the practical realization of large Non-Orthogonal systems.

The recently proposed MultiSphere SD framework enables practical and low latency massively parallel processing for large MIMO systems [19]. MultiSphere focuses the available processing power on the most "promising" vector solutions. To achieve this a "Metric-of-Promise"(MoP) is introduced that exploits the MIMO interference matrix to identify the Relative Position Vectors (RPVs) of the most promising solutions prior to detection. These RPVs are identified by an approximate SD tree search that can be realized by means of a K-Best approach with $\mathrm{K}$ being the number of available Parallel Elements (PEs) as suggested in [19]. These RPVs are demapped to symbols based on their Euclidean distance to the received vector.

While MultiSphere can efficiently parallelize the large MIMO detection problem, it focuses on the hard detection case and on full rank systems. NorthCore extend this framework to soft detection and makes it applicable to any NonOrthogonal scheme. Still, for soft detection a larger number of candidate solutions needs to be examined than in the case of hard detection, since the corresponding soft information calculation consists of multiple constrained hard detection problems [8]. Due to this, MultiSphere's preprocessing, which has been proposed to be based on the K-Best SD, becomes of high complexity due to the required large $\mathrm{K}$ values and the corresponding sorting operations. NorthCore introduces a new preprocessing approach that resolves these bottlenecks. In particular the number of examined nodes are different for each $\mathrm{SD}$ layer and the $\mathrm{K}$ value is adaptively based on the specific interference matrix realization, in contrast to the traditional approaches where all constellation nodes are examined and the value of $\mathrm{K}$ is set based on the worst case transmission condition. In addition, the extensive sorting operations are now unnecessary, substantially reducing the preprocessing computational complexity.

The rest of the paper is structured as follows. In Section II we introduce a generic model to describe Non-Orthogonal systems. In Section III we provide a primer on SphereDecoding-based detection for Non-Orthogonal systems, and in Section IV we describe NorthCore; the proposed massively parallel detection scheme, together with the improved preprocessing method. Finally, in Section $\mathrm{V}$ we evaluate NorthCore for decoding of LDS-OFDM and SEFDM signals. Complexity savings of up to an order of magnitude are shown when comparing NorthCore with state-of-the-art detectors for SEFDM transmissions. We also show that, when NorthCore is applied to the detection of sparse LDS-OFDM signals, it can reduce both complexity and latency by more than an order of magnitude compared to MPA while providing improved throughput.

\section{GENERIC NON-ORTHOGONAL TRANSMISSION MODELING}

The baseband received signal for a Non-Orthogonal system can be given by

$$
\mathbf{y}=\mathbf{H s}+\mathbf{w}
$$

where $\mathbf{y}$ is the $N \times 1$ received vector, $\mathbf{s}$ is the $M \times 1$ transmitted symbol vector with elements belonging to a constellation $\mathcal{O}$, w is the $M \times 1$ additive white Gaussian noise vector with variance $\sigma^{2}$ and $\mathbf{H}$ is the interference matrix that differs per Non-Orthogonal system.

LDS-OFDM NOMA systems: In an LDS-OFDM system an (orthogonal) subcarrier is loaded with the signals of multiple users which are superimposed. The $N \times 1$ received signal vector for an LDS-OFDM system where $N$ orthogonal subcarriers are occupied by $M$ users is given by

$$
\mathbf{y}=\left[\mathbf{h}_{1}, \mathbf{h}_{2}, \ldots \ldots, \mathbf{h}_{M}\right] \cdot\left[\mathbf{g}_{1}, \mathbf{g}_{2}, \ldots \ldots, \mathbf{g}_{M}\right] \mathbf{s}+\mathbf{w}
$$

where $\mathbf{h}_{k}$ is the frequency domain channel for user $k$ and $\mathrm{g}_{k}$ is the "sparse signature vector" for the Non-Orthogonal user $k$, which consists of complex entries that define how the signal is spread over subcarriers [1]. These sparse signature vectors are selected by predefined codebooks as discussed in [20]. Therefore, in relation to (1) the interference matrix $\mathbf{H}_{L D S-O F D M}=\left[\mathbf{h}_{1}, \mathbf{h}_{2}, \ldots \ldots, \mathbf{h}_{M}\right] \cdot\left[\mathbf{g}_{1}, \mathbf{g}_{2}, \ldots \ldots, \mathbf{g}_{M}\right]$, and it is rank deficient since $N<M$.

SEFDM systems: An SEFDM [5] block consists of $M$ complex symbols transmitted within a time period $T$. Each of these $M$ complex symbols modulate a Non-Orthogonal subcarrier. The bandwidth compression factor $\alpha$ is defined as $\alpha=\Delta f T$, with $\Delta f$ being the frequency spacing between subcarriers, and with $\alpha=1$ corresponding to an orthogonal system (e.g., OFDM). Then, the $N \times 1$ received vector, consisting of the received signal at each Non-Orthogonal subcarrier, is given by

$$
\mathbf{y}=\mathbf{B}^{H} \mathbf{C}_{h} \mathbf{F}_{\alpha} \mathbf{s}+\mathbf{w},
$$

where the $M \times M$ fractional IFFT matrix $\mathbf{F}_{\alpha}$ consists of the entries $F_{\alpha}[k, n]=\exp (j 2 \pi \alpha(k-1)(n-1) / M) / \sqrt{M}$ for $n, k=1, \ldots, M$. The $M \times M$ matrix $\mathbf{C}_{h}$ is circulant with its first column being $\left[h_{0}, h_{1}, \ldots, h_{L-1}, 0, \ldots, 0\right]^{T}$ and $L$ being equal to the number of channel taps in the time domain. Matrix $\mathbf{B}$ represents an orthonormal base $\mathbf{B}=\left[b_{1}, \ldots, b_{M}\right]$ which spans the SEFDM signal space and could be computed using a Gram-Schmidt orthonormalisation procedure as in [21]. Consequently, the SEFDM interference matrix is $\mathbf{H}_{S E F D M}=\mathbf{B}^{H} \mathbf{C}_{h} \mathbf{F}_{\alpha}$, and is ill-determined [13].

\section{SPHERE DECODING FOR NON-ORTHOGONAL SYSTEMS}

As discussed in Section II, the interference matrix $\mathbf{H}$ could be ill-determined or rank deficient. Tikhonov regularization [22] is a proven method for mitigating the effects of small eigenvalues of an ill-determined rank matrix and has also been applied to rank deficient systems [23]. In particular, instead of 
performing a $\mathrm{QR}$ decomposition on $\mathbf{H}$ we employ the $\mathrm{QR}$ decomposition of [24] on the Tikhonov regularised matrix $\overline{\mathbf{H}}$

$$
\overline{\mathbf{H}} \triangleq\left[\begin{array}{c}
\mathbf{H} \\
\lambda \mathbf{I}_{M}
\end{array}\right]=\overline{\mathbf{Q}} \overline{\mathbf{R}}=\left[\begin{array}{l}
\mathbf{Q}_{1} \\
\mathbf{Q}_{2}
\end{array}\right] \overline{\mathbf{R}},
$$

with the regularisation parameter $\lambda=\sigma / \mathbf{E}|s|$. Where $\overline{\mathbf{Q}}$ is a $2 N \times N$ orthonormal matrix and $\overline{\mathbf{R}}$ is a $N \times N$ upper triangular matrix. Then, the "hard" $\mathrm{ML}$ estimation problem can be expressed as

$$
\hat{\mathbf{s}}_{M L}=\arg \min _{\mathbf{s} \in \mathcal{O}^{M}}\left\{\|\widetilde{\mathbf{y}}-\overline{\mathbf{R}} \mathbf{s}\|^{2}-\lambda^{2}\|\mathbf{s}\|^{2}\right\},
$$

where $\widetilde{\mathbf{y}}=\mathbf{Q}_{1}^{H} \mathbf{y}$ is an $M \times 1$ vector. Since $\overline{\mathbf{R}}$ is an upper triangular matrix, finding the ML solution can be translated into a tree search of height $M$ and branching factor $|\mathcal{O}|$. Each node at level $l$ can be identified by its partial symbol vector $\mathbf{s}_{l}=\left[s_{l}, s_{l+1}, \ldots, s_{M}\right]$ which also determines, the path from the root to that node, as well as from its partial Euclidean distance (PD) which can be calculated recursively as $d\left(\mathbf{s}_{l}\right)=$ $d\left(\mathbf{s}_{l+1}\right)+e\left(s_{l}\right)$ where $e\left(s_{l}\right)$ is the non-negative cost assigned to each branch,

$$
e\left(s_{l}\right)=\left(\left|\tilde{y}_{l}-\sum_{k=l}^{M} \bar{R}_{l k} s_{l}\right|^{2}+\lambda^{2}\left(E_{s_{\max }}-\left|s_{l}\right|^{2}\right)\right) .
$$

Here, $E_{s_{\max }}=\max \left(|s|^{2}\right)$ is the maximum energy of symbols chosen from the constellation $\mathcal{O}$. Then the ML detection problem is equivalent to finding the vector $\mathbf{s}$ with minimum $d\left(\mathbf{s}_{1}\right)$. According to the Schnorr-Euchner (SE) [25] enumeration the nodes are visited in an ascending order of their $e\left(s_{l}\right)$. For non constant amplitude transmit symbol constellations the minimization problem in (5) differs from the traditional SD tree search in [9], [19], [26] due to the $\lambda^{2}\left(E_{s_{\max }}-\left|s_{l}\right|^{2}\right)$ term. As a result, applying the SE enumeration without exhaustively calculating the PDs of all constellation symbols, and instead by using simple geometrical properties as in [9], is not anymore feasible. To cope with this problem enumeration schemes similar to [27], [28] could be used. However, these techniques are highly sequential and unsuitable for parallel processing. In rest of the paper we will discuss how we can efficiently cope with this issue in massively parallel detection approaches. In practical systems that employ soft channel decoding approaches like LDPC, soft information is required. The computation of soft information in the form of $\mathrm{Log}$ Likelihood Ratios (LLRs), when the Max-Log approximation is employed, involves multiple constrained ML searches [8]. In particular, the LLR for the $j$ th coded bit $b_{j}$ could be expressed as

$$
\begin{aligned}
L\left(b_{j}\right) \approx & \min _{\mathbf{s} \in S_{j}^{-1}}\left\{\frac{1}{\sigma^{2}}\|\tilde{\mathbf{y}}-\overline{\mathbf{R}} \mathbf{s}\|^{2}+\frac{\lambda^{2}}{\sigma^{2}}\left(E_{s_{\max }}-\|\mathbf{s}\|^{2}\right)\right\} \\
& -\min _{\mathbf{s} \in S_{j}^{+1}}\left\{\frac{1}{\sigma^{2}}\|\tilde{\mathbf{y}}-\overline{\mathbf{R}} \mathbf{s}\|^{2}+\frac{\lambda^{2}}{\sigma^{2}}\left(E_{s_{\max }}-\|\mathbf{s}\|^{2}\right)\right\} \\
= & \operatorname{sign}\left(x_{j}\right)\left(D_{j}^{\overline{M L}}-D^{M L}\right),
\end{aligned}
$$

where $x_{j}$ is the $j$ th entry of the ML solution's bit mapping and $S_{j}^{-1}, S_{j}^{+1}$ are the subsets of possible symbol vectors with $j$ th bipolar bit set to $-1,+1$ respectively. Here $D^{M L}$ is the metric of the ML solution and $D_{j}^{\overline{M L}}$ is the minimum metric from subset $S_{j}^{\overline{x_{j}}}$ (counter hypothesis [10]) for bit $j$.

\section{NORTHCORE'S DESIGN}

Originally, the MultiSphere [19] framework targeted the "hard" ML problem. MultiSphere focuses the available processing power on the most "promising" SD tree paths to constitute the transmitted symbol vector. This is achieved by a (prior to the detection stage), preprocessing stage that, based on the specific channel realization, identifies the most promising tree paths to include the transmitted symbol vector. The likelihood of each tree path is characterized by a heuristic metric of promise $(\mathrm{MoP}) \mathcal{M}$. The most promising tree paths could be identified by an approximate K-Best SD tree search with $K$ being the maximum number of examined candidate solutions as suggested in [19]. Then, during detection, a demapping procedure is used to de-map these paths to actual symbol vectors based on their Eucldean distances to the received signal. However, as discussed in Section I, MultiSphere does not directly apply to Non-Orthogonal systems as the proposed MoP does not account for the effect of interference matrix regularization that is needed to account for the illdetermined properties of the Non-Orthogonal systems. In Section IV-A a similar MoP to [19] is introduced for generic NonOrthogonal systems that utilize regularized QR decomposition (Section IV-A). Moreover, a larger number of tree paths (e.g., candidate solutions) needs to be processed in parallel in "soft" detection systems than in "hard" detection systems, due to the corresponding multiple constrained ML searches (see Section III). Then, the originally proposed K-Best-based preprocessing (with $K$ being equal to the number of examined tree paths) can become impractical since it requires sorting operations of a complexity of $O(K|\mathcal{O}| \log \{K|\mathcal{O}|\})$ and metric calculations of a complexity of $O(K|\mathcal{O}|)$ per SD level. In Section IV-B we introduce a novel efficient preprocessing module which improves on the regular K-Best approach by considering a lower number of candidates $(\tilde{K} \leq K)$ that are chosen adaptively, based on the specific interference matrix realization. The extensive sorting operations required for KBest SD are avoided by a new threshold based selection which requires only a fraction of the sorting comparisons (Section IV-B). In Section V we show that the proposed preprocessing approach can be up to an order of magnitude less complex without any significant performance loss.

Similarly to MultiSphere, the preprocessing stage of NorthCore finds the most promising tree paths by means of ordered distances to the received points. During the detection stage a procedure is required to demap those paths onto actual symbols. In Section IV-C we show that MultiSphere's tree path to symbol de-mapping procedure [19] can still, be approximately been used by NorthCore, despite the modified metric introduced in (6). 


\section{A. NorthCore's MoPs}

Similar to [19], a tree path is described by means of its ordered (in terms of PDs) position of its nodes to the received observable by an $M \times 1$ relative position vector $(\mathrm{RPV}) \mathrm{k}$, with integer elements $k_{l}\left(l \in[1, M]\right.$ and $\left.k_{l} \in[1,|O|]\right)$. Then, for the corresponding tree path, the node at level $l$ is the $k_{l}^{t h}$ closest node to the received observable $\tilde{y}_{l}$. Due to the regularization of the interference matrix, the received vector $\widetilde{\mathbf{y}}$ includes a residual self-interference term that together with the noise [24] they form an effective noise term

$$
\overline{\mathbf{w}}=\mathbf{Q}_{1}^{H} \mathbf{w}-\lambda \mathbf{Q}_{2}^{H} \mathbf{s} .
$$

By approximating this noise as Gaussian, and similarly to [19], the MoPs $(\mathcal{M}(\mathbf{k}))$ for for Non-Orthogonal systems can be recursively calculated as

$$
\mathcal{M}\left(\mathbf{k}_{l}\right)=\mathcal{M}\left(\mathbf{k}_{l+1}\right)+\left(k_{l}-1\right)\left|\bar{R}_{l l}\right|^{2},
$$

where $\mathbf{k}_{l}$ is the partial RPV and $\mathcal{M}\left(\mathbf{k}_{l}\right)$ is the partial MoP of $\mathrm{k}$ at level $l$.

\section{B. NorthCore's preprocessing stage}

The purpose of the preprocessing stage is to identify the most promising RPVs. To reduce the search space of the preprocessing stage we consider a threshold for the MoPs that determines the number of required RPVs $\tilde{K}$. The number of required RPVs $(\tilde{K} \leq K)$ depend on the specific interference matrix realization and correspond to the number of required PEs. To achieve this we only consider the $\tilde{K}$ RPVs that satisfy

$$
\mathcal{M}(\mathbf{k}) \leq \mathcal{M}_{t h}
$$

The threshold $\mathcal{M}_{t h}$ is a function of $\sigma^{2}$. For an example the threshold $\mathcal{M}_{t h}=4 \sigma^{2}$ is considered in Section V. Since the recursive structure of the MoP calculation in (9) resembles that of an SD, the search for RPVs translates into a tree search. Therefore the RPVs with the smallest MoPs are identified by a K-Best SD like tree search where each node at level $l$ is characterized by a partial RPV $\mathbf{k}_{l}$ and a partial MoP $\mathcal{M}\left(\mathbf{k}_{l}\right)$ similar to the description in Section III. However, as we will explain later, our approach results in a different $\tilde{K}$ value per tree level and does not require any sorting operations.

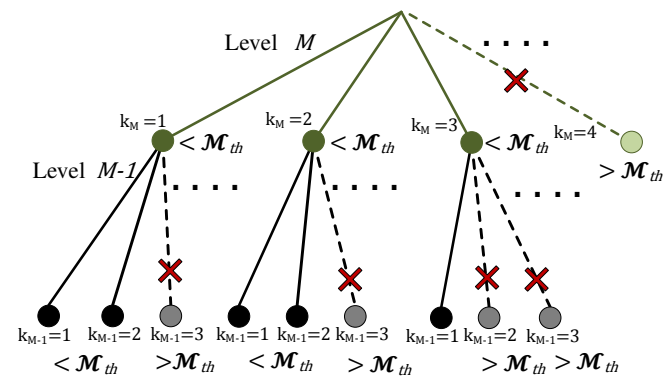

Fig. 1: Tree path selection example.

The selection of the most promising RPVs starts at the highest tree level. Then, the tree nodes are visited in the ascending order of their $K_{M}$ indices and all the nodes with a partial MoP larger than $\mathcal{M}_{t h}$ are pruned. For each of the survived nodes at level $M$, the child node with the smallest partial MoP is expanded first. At level $M-1$ the expanded nodes are visited in an ascending order of their parent's partial MoPs and all those with a partial MoP larger than $\mathcal{M}_{t h}$ are pruned. The approach continues with expanding the child node with the second partial MoP for each of the survived nodes at level $M$. Those nodes with partial MoPs larger than $\mathcal{M}_{t h}$ are also pruned. The node expansion continues until either all nodes at level $M-1$ are examined or until when the number of non-pruned nodes reaches $K$. Then, the same procedure is applied for the rest of the tree levels. An example of the proposed MoP identification method is shown in Fig. 1. The process starts at level $M$. First, we compare the partial MoPs of all nodes with the probabilistic threshold $\mathcal{M}_{t h}$. Where the fourth node $\left(\mathbf{k}_{M}=4\right)$ is pruned since we assume that the corresponding partial MoP exceeds the probabilistic threshold. Then only three partial RPVs $\left(\mathbf{k}_{M}=1,2,3\right)$ remain as survivors for the next level (level $M-1$ ). At level $M-1$, the first child node for each survived node is expanded. Then, all expanded nodes are selected since their MoPs are within the threshold. Next, the second child node for each of the survived nodes at level $M$ is expanded. In this case, the second child node of the third parent node (partial RPV $\mathbf{k}_{M-1}=[2,3]^{T}$ ) is not selected since we assume that its partial metric exceeds $\mathcal{M}_{t h}$. Subsequently, the third child node for each of the survived nodes at level $M$ is expanded, and those with partial MoPs larger than $\mathcal{M}_{t h}$ are again pruned. Since all other nodes at level $M-1$ have been pruned, the process will continue by expanding the first child node of these survived nodes (partial RPVs $\left.\mathbf{k}_{M-1}=[1,1]^{T},[1,2]^{T},[2,1]^{T},[2,2]^{T},[1,3]^{T}\right)$.

\section{NorthCore's de-mapping}

The preprocessing stage finds the most promising paths (RPVs) by means of ordered distances to the received point $\hat{y}_{l}$, where

$$
\hat{y}_{l}=\left(\tilde{y}_{l}-\sum_{j=l+1}^{n_{t}} \bar{R}_{l j} s_{j}\right) \bar{R}_{l l}^{-1} .
$$

During the detection stage we need to demap these nodes onto actual symbols. This would traditionally require exhaustively calculating the corresponding distances for all symbols and sorting them, that would result in a substantial complexity overhead. Instead, MultiSphere [19] introduced a symbol mapping of two-dimensional zigzag coordinates. Then, an approximate symbol ordering relative to $\hat{y}_{l}$, could be predefined as a sequence of these two-dimensional zigzag coordinates [19]. Following the same principles we utilize the same preordering introduced in [19]. We note, that based on (6), the actual ordering should take place based on the corresponding partial distance $e\left(s_{l}\right)$ and not on the distance from the received symbol. However, this is feasible since the second part in (6) has been modelled as additional noise (see Eq. 8). 


\section{Simulation Evaluation}

First we evaluate the performance of NorthCore in LDSOFDM systems in 4 tap i.i.d Rayleigh fading channels. Fig. 2 compares the BER of NorthCore to this of MPA for an LDS-OFDM system where 6 users utilize 4 subcarriers (SCs). The sparse signature matrix and the codebooks of [20] have been adopted. It can been seen that, for thos scenario, NorthCore can provide an SNR gain of nearly $2 \mathrm{~dB}$ compared to traditional MPA. For this signature matrix and interfering users the computation complexity of MPA is of $N|\mathcal{O}|^{3}$ per iteration, while NorthCore requires only a PD calculation per visited node similarly to SFSD [11]. In particular, and as shown in Fig. 2, NorthCore requires 5832 complex multiplications [11] while MPA requires 16384 complex multiplications per iteration, resulting in more than an order of magnitude reduction in overall complexity. In addition, in contrast to MPA, NorthCore does not require any iterations, and therefore it has a detection latency similar to this of the highly suboptimal linear detection approaches.

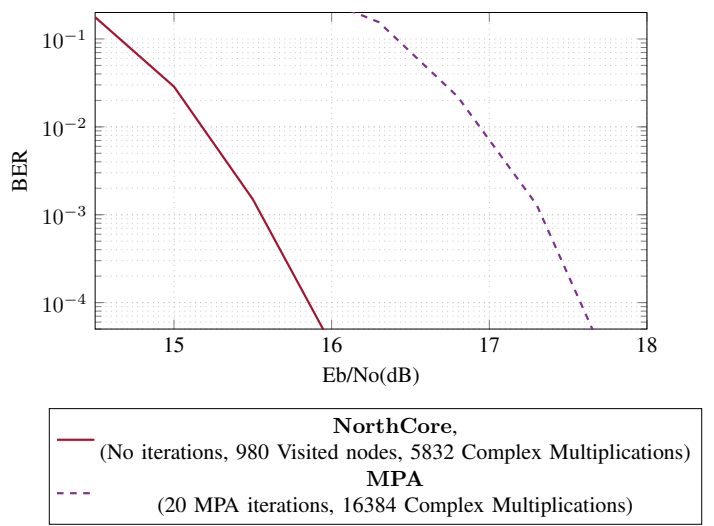

Fig. 2: BER of NorthCore in comparison with MPA for LDS OFDM,

Fig. 3a shows the average corresponding complexity/performance trade-off between the NorthCore's preprocessing and the traditional MultiSphere's K-Best approach. In particular, it shows that an order of magnitude reduction can be achieved for a very small BER performance loss.

Next we apply NorthCore to SEFDM systems, and we compare it to state-of-the-art SEFDM detectors in 4 tap i.i.d Rayleigh fading channels. In particular, Fig. 4 shows the soft detection complexity requirements (in terms of visited nodes) and the BER performance of NorthCore compaerd to SFSD, soft K-Best SD [11], [12] and the soft FFT detector of [15], for 16 subcarrier (SC) systems with $\alpha=0.67$ and 0.6 . It can be seen that NorthCore's complexity gains increase when the overlap between subcarriers increases (when the parameter $\alpha$ decreases) and it NorthCore can be up to an order of magnitude less complex than the state-of-the-art when $\alpha=0.6$.

Fig. 5a shows the the complexity savings of NorthCore's preprocessing in comparison to the MultiSphere's K-Best approach for the SEFDM system considered in Fig. 4. Again, the proposed preprocessing reduces complexity by an order of magnitude while the corresponding performance loss is small.

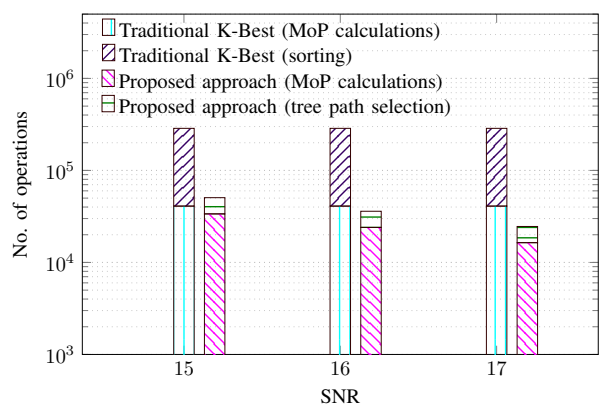

(a)

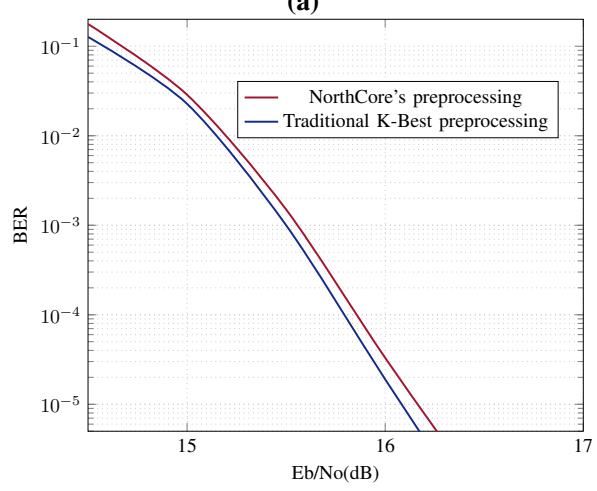

(b)

Fig. 3: a) Complexity of NorthCore's preprocessing in comparison to MultiSphere's K-Best approach, b) the corresponding BER performance, for a 6 user LDS-OFDM systems employing 16 point codebooks.

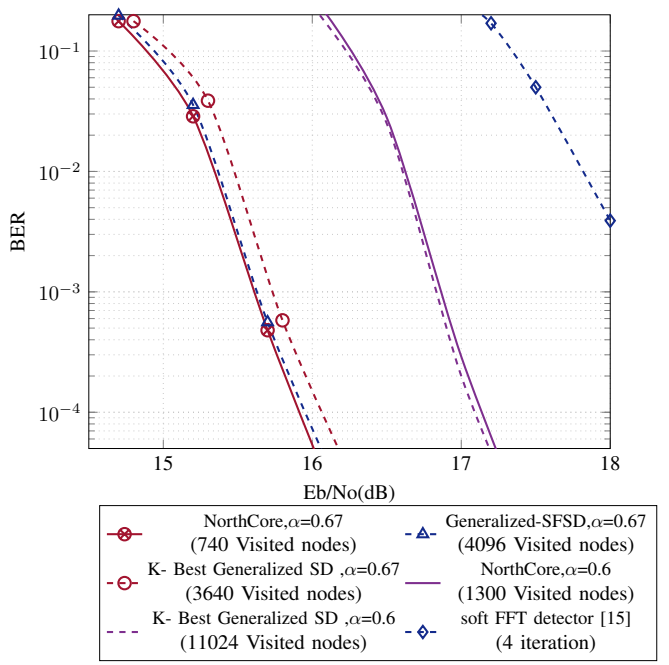

Fig. 4: Soft detection BER performance of NorthCore in comparison with existing methods for a 16 QAM 16 subcarrier SEFDM system with $K=128$. Rayleigh fading channels and $1 / 2$ rate LDPC codes are assumed.

\section{Conclusions}

This work presents some first results towards a massively parallel detection framework for Non-Orthogonal systems with nearly an order of magnitude complexity reductions compared to existing approaches. Future work will focus on generalizing these results to additional non-orthogonal signal transmissions 


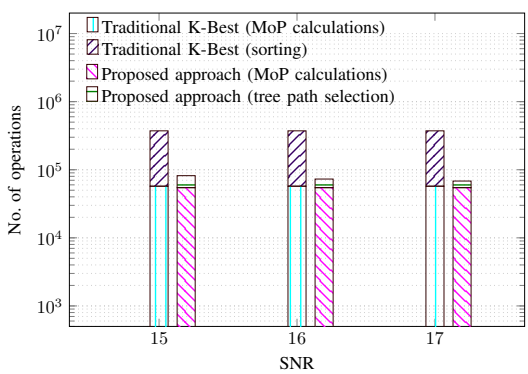

(a)

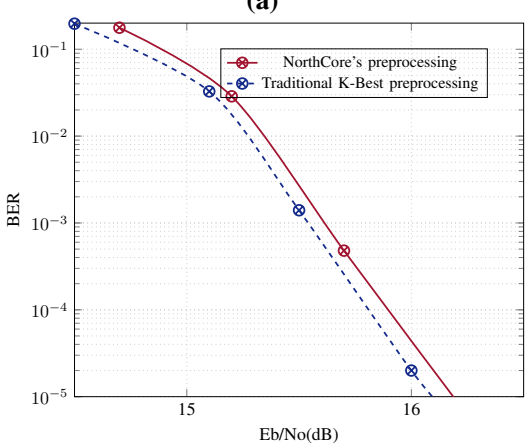

(b)

Fig. 5: a) Complexity of NorthCore's preprocessing in comparison to MultiSphere's K-Best approach, b) The corresponding BER performance, for 16 SC SEFDM systems with $\alpha=0.67$ and $K=128$.

and combinations of those.

\section{ACKNOWLEDGMENT}

The research leading to these results has been supported from the UK's Engineering and Physical Sciences Research Council (EPSRC Grant EP/M029441/1). The Authors would like to also thank the members of University of Surrey 5GIC (http://www.surrey.ac.uk/5GIC) for their support.

\section{REFERENCES}

[1] R. Hoshyar, R. Razavi, and M. Al-Imari, "LDS-OFDM an efficient multiple access technique," in IEEE VTC, May 2010, pp. 1-5.

[2] K. Au, L. Zhang, H. Nikopour, E. Yi, A. Bayesteh, U. Vilaipornsawai, J. Ma, and P. Zhu, "Uplink contention based scma for $5 \mathrm{~g}$ radio access,' in IEEE GC Wkshps, Dec 2014.

[3] J. E. Mazo, "Faster-than-nyquist signaling," The Bell System Technical Journal, vol. 54, no. 8, pp. 1451-1462, Oct 1975.

[4] F. Rusek and J. B. Anderson, "Constrained capacities for faster-thannyquist signaling," IEEE Trans. Inf. Theory, vol. 55, no. 2, pp. 764-775, Feb 2009.

[5] I. Kanaras, A. Chorti, M. R. D. Rodrigues, and I. Darwazeh, "Spectrally efficient FDM signals: Bandwidth gain at the expense of receiver complexity," in Proc. of IEEE ICC, June 2009, pp. 1-6.

[6] R. Razavi, M. AL-Imari, M. A. Imran, R. Hoshyar, and D. Chen, "On receiver design for uplink low density signature OFDM (LDS-OFDM),' IEEE Trans. on Commun., vol. 60, no. 11, pp. 3499-3508, November 2012.

[7] L. Dai, B. Wang, Y. Yuan, S. Han, C. 1. I, and Z. Wang, "Non-orthogonal multiple access for $5 \mathrm{~g}$ : solutions, challenges, opportunities, and future research trends," IEEE Commun. Magazine, September 2015.

[8] C. Studer, A. Burg, and H. Bolcskei, "Soft-output sphere decoding: algorithms and vlsi implementation," IEEE J. Sel. Areas Commun., vol. 26, no. 2, pp. 290-300, February 2008.

[9] K. Nikitopoulos, J. Zhou, B. Congdon, and K. Jamieson, "Geosphere: Consistently turning MIMO capacity into throughput," in Proc. of ACM SIGCOMM, 2014, pp. 631-642.
[10] C. Studer, A. Burg, and H. Bölcskei, "Soft-output sphere decoding: algorithms and VLSI implementation," IEEE J. Sel. Areas Commun., vol. 26, pp. 290-300, 2008.

[11] L. G. Barbero and J. S. Thompson, "Extending a fixed-complexity sphere decoder to obtain likelihood information for turbo-mimo systems," IEEE Trans. Veh. Technol., vol. 57, no. 5, pp. 2804-2814, Sept 2008.

[12] Z. Guo and P. Nilsson, "Algorithm and implementation of the k-best sphere decoding for mimo detection," IEEE JSAC, vol. 24, no. 3, pp. 491-503, March 2006.

[13] S. Isam, I. Kanaras, and I. Darwazeh, "A truncated SVD approach for fixed complexity spectrally efficient FDM receivers," in Proc. of IEEE WCNC, March 2011, pp. 1584-1589.

[14] T. Xu and I. Darwazeh, "M-QAM signal detection for a non-orthogonal system using an improved fixed sphere decoder," in Proc. CSNDSP, July 2014, pp. 623-627.

[15] — "A soft detector for Spectrally Efficient Systems with NonOrthogonal overlapped sub-carriers," IEEE Commun. Lett, vol. 18, no. 10 , pp. 1847-1850, Oct 2014.

[16] H. Huawei, "Candidate schemes for superposition transmission," 3GPP, Tech. Rep. R1-152493, May 2015.

[17] P. Popovski et al., "Requirement analysis and design approaches for 5g air interface," METIS Project, Tech. Rep. ICT-317669-METIS/D2.1, Aug 2013.

[18] R. Courtland, "Transistors could stop shrinking in 2021," IEEE Spectrum, vol. 53, no. 9, pp. 9-11, 2016.

[19] K. Nikitopoulos, D. Chatzipanagiotis, C. Jayawardena, and R. Tafazolli, "Multisphere: Massively parallel tree search for large sphere decoders," in Proc. of IEEE GLOBECOM, 2016.

[20] J. Bao, Z. Ma, Z. Ding, G. K. Karagiannidis, and Z. Zhu, "On the design of multiuser codebooks for uplink SCMA systems," IEEE Commun. Lett, vol. 20, no. 10, pp. 1920-1923, Oct 2016.

[21] A. Chorti, I. Kanaras, M. R. D. Rodrigues, and I. Darwazeh, "Joint channel equalization and detection of spectrally efficient fdm signals,' in Proc. IEEE PIMRC, Sept 2010, pp. 177-182.

[22] P. C. Hansen, "Truncated singular value decomposition solutions to discrete ill-posed problems with ill-determined numerical rank," SIAM Journal on Scientific Computing, vol. 11, no. 3, pp. 503-518, 1990.

[23] T. Cui and C. Tellambura, "An efficient generalized sphere decoder for rank-deficient MIMO systems," in Proc. of IEEE VTC, vol. 5, Sept 2004 pp. 3689-3693 Vol. 5 .

[24] D. Wubben, R. Bohnke, V. Kuhn, and K. D. Kammeyer, "MMSE extension of V-BLAST based on sorted QR decomposition," in Proc. of IEEE VTC, vol. 1, Oct 2003, pp. 508-512 Vol.1.

[25] A. Burg, M. Borgmann, M. Wenk, M. Zellweger, W. Fichtner, and H. Bolcskei, "VLSI implementation of MIMO detection using the sphere decoding algorithm," IEEE J. of Solid-State Circ., vol. 40, no. 7, pp. 1566-1577, 2005.

[26] C. Husmann, G. Georgis, K. Nikitopoulos, and K. Jamieson, "FlexCore: Massively parallel and flexible processing for large MIMO access points," in Proc. of USENIX NSDI, 2017, pp. 197-211.

[27] C. H. Liao, I. W. Lai, K. Nikitopoulos, F. Borlenghi, D. Kammler, and M. Witte, "Combining orthogonalized partial metrics: Efficient enumeration for soft-input sphere decoder," in Proc. IEEE PIMRC, Sept 2009, pp. 1287-1291.

[28] K. Nikitopoulos, D. Zhang, I. w. Lai, and G. Ascheid, "Complexityefficient enumeration techniques for soft-input, soft-output sphere decoding," IEEE Commun. Lett., vol. 14, no. 4, pp. 312-314, April 2010. 